\title{
New formulation of the beam propagation method based on the slowly varying envelope approximation
}

\author{
H.J.W.M. Hoekstra, G.J.M. Krijnen and P.V. Lambeck \\ MESA-Research Institute, University of Twente, P.O. Box 217, 7500 AE Enschede, Netherlands
}

Received 27 November 1992

\begin{abstract}
We combine the theoretical results of some recent publications on the beam propagation method based on the finite difference method (FDBPM) and the slowly varying envelope approximation (SVEA). The use of the SVEA leads to a considerable reduction of the computation time but introduces a fundamental error in the phase velocity of a given mode and also to an error due to the finite step size along the propagation direction. A new, effective scheme is given, which results in a considerable reduction of these errors, without much increase of the computational effort. An error analysis and a few results of applications are given.
\end{abstract}

\section{Introduction}

Efficient and accurate beam propagating methods are of importance for simulations on structures for which no analytical solutions exist and also for the testing of the validity of approximate analytical expressions. Introducing the SVEA [1] the BPM wins in computation speed but looses accuracy if modes with a large difference in the mode index are involved [2]. Recent publications [2,3] show that the accuracy can be improved considerably by using a perturbation expansion.

It is the aim of this paper to show that further improvements can be obtained starting from the results of both refs. [2] and [3]. Expressions will be given for the second order perturbation expansion in 2D, assuming TE polarisation. However, the method is quite general and can be extended to higher order, other polarisations, to $3 \mathrm{D}$ and to vectorial implementations. An error analysis of the method and a few results of its application will be given.

\section{Theory}

Assuming a time dependence $\exp (\mathrm{i} \omega t)$ the field, $E(x, z)$, propagating mainly in the positive $z$ direction, can be written as
$E(x, z) \equiv \psi(x, z) \exp \left(-\mathrm{i} k_{0} n_{0} z\right)$.

Here $n_{0}$ is a suitably chosen mean refractive index. Substitution of eq. (1) into the wave equation and also introducing a discretisation along the $x$-axis leads to

i $a \partial \psi / \partial z=\mathbf{M} \psi+\partial^{2} \psi / \partial z^{2}$.

Here $a=2 k_{0} n_{0}$ and $\mathbf{M}$ is a tridiagonal matrix corresponding to the operator, $O \equiv \partial^{2} / \partial x^{2}+$ $\left.k_{0}^{2}\left[n^{2}(x, z)-n_{0}^{2}\right)\right]$. In the SVEA the last term of eq. $(2)$ is usually neglected. This term can be taken into account quite effectively by using the recursion relation [3]:

$\left.\frac{\partial}{\partial z}\right|_{n}=\frac{-\mathrm{iM} / a}{1+(\mathrm{i} / a) \partial /\left.\partial z\right|_{n-1}}$.

For $n=4$ this leads to a Padé $(2,2)$ expression [3]:

$\frac{\partial \psi}{\partial z}=\frac{-\mathrm{i} \mathbf{M} / a-\mathrm{i} 2 \mathbf{M}^{2} / a^{3}}{1+3 \mathbf{M} / a^{2}+\mathbf{M}^{2} / a^{4}} \boldsymbol{\psi} \equiv \mathrm{iA} \psi$.

For the calculation of the propagated field after a step, $\Delta z$, it is of importance to use, on integrating eq. (4) between $s \Delta z$ and $(s+1) \Delta z$, a kind of extended Crank-Nicolson scheme [2]. Then, taking into account the exponential nature of the solution of eq. (4), expressing $\psi^{s+1}$ in terms of $\psi^{s}$ and using a Taylor expansion for the exponent it follows 


$$
\begin{aligned}
& \left(\mathrm{I}-\mathrm{iA} \Delta z / 2-\mathbf{A}^{2} \Delta z^{2} / 12\right) \boldsymbol{\psi}^{s+1} \\
& =\left(\mathbf{I}+\mathrm{iA} \Delta z / 2-\mathbf{A}^{2} \Delta z^{2} / 12\right) \boldsymbol{\psi}^{s} .
\end{aligned}
$$

Here $I$ is the unit matrix.

Collecting terms up to $\mathbf{M}^{2}$ it follows for the matrix equation to be solved:

$\mathbf{Q}_{+} \boldsymbol{\psi}^{s+1}=\mathbf{Q}_{-} \boldsymbol{\psi}^{s}$,

with

$$
\begin{aligned}
\mathbf{Q}_{ \pm} & =\mathrm{I}+\left(3 / a^{2} \pm \mathrm{i} / a^{4}\right) \mathbf{M} \\
& +\left[ \pm \mathrm{i} \Delta z / a^{3}+1 / a^{4}-\Delta z^{2} /\left(12 a^{2}\right)\right] \mathbf{M}^{2} .
\end{aligned}
$$

$\psi^{s+1}$ can be solved from eq. (6) using twice the procedure for solving a tridiagonal-matrix equation, as one can write

$\mathbf{Q}_{ \pm}=c_{0, \pm}\left(\mathbf{M}-c_{1, \pm} \mathbf{I}\right)\left(\mathbf{M}-c_{2, \pm} \mathbf{I}\right)$,

where $c_{l}(l=0,1,2)$ are complex constants which depend only on $n_{0}, k_{0}, \Delta x$ and $\Delta z$.

The inaccuracy of the method can be expressed exactly by using the same approach as described in ref. [2]. We consider a propagating modal field field with a mode index $n_{e}$, differing from $n_{0}$. Defining $\delta \equiv$ $\left(n_{\mathrm{e}} / n_{0}-1\right)$, and $k^{\prime} \equiv k_{0} n_{0} \Delta z$ it follows for the error in the effective index, $\Delta n_{e}$, of the propagated, discretesized modal field:

$\arctan \left[2 p q /\left(p^{2}-q^{2}\right)\right]=k^{\prime}\left(\Delta n_{\mathrm{e}} / n_{0}+\delta\right)$,

with $p \equiv 1+3 f+f^{2}-k^{\prime 2} f^{2} / 3$ and $q \equiv k^{\prime} f+2 k^{\prime} f^{2}$. Here we have used that $\mathbf{M} \psi=k_{0}^{2}\left(n_{\mathrm{e}}^{2}-n_{0}^{2}\right) \psi$ for the modal field $\psi$, and $f \equiv\left(n_{\mathrm{e}}^{2}-n_{0}^{2}\right) /\left(2 n_{0}\right)^{2}=\delta / 2+$ $\delta^{2} / 4$. From eq. (8) it follows for the relative error:

$\Delta n_{\mathrm{e}} / n_{0} \approx-0.06 \delta^{5}-0.04 k^{2} \delta^{4}$.

It is straightforward to extend the presented theory to higher-order accuracy be extension of both eqs. (4) and (5).

\section{Results}

For the simulations we have used the interface conditions according to ref. [4] and the transparent boundary conditions (TBC) of ref. [5]. However, we have found that for some problems the application of the TBC leads to unphysical gain at the boundaries. In order to prevent this we have put in our program some extra constraints on the quantity $k_{x} \Delta x$, defined by

$\exp \left(i k_{x} \Delta x\right)=\psi_{m} / \psi_{m+1}$.

Here we consider only the left boundary, the right boundary is treated similarly. We assume that $m=0$ corresponds to the left boundary, then, inspecting eqs (6) and ( 7 ) it follows that $m \geqslant 2$ for the second order case. The magnitude of $m$ is selected according to ref. [5]. Besides the restrictions on the real part of $k_{x}$ [5] we put the imaginary part $k_{x} \Delta x$ slightly larger than zero $(\approx 0.01)$ if this quantity is negative. After $k_{x}$ has been reset, if necessary, the fields at the boundary, $\psi_{0}$ and $\psi_{1}$, can be expressed in terms of $\psi_{2}$, which is needed for the propagation step. After this propagation step $k_{x}$ is recalculated using $m=2$ and reset, if needed, as above. Then, the newly defined ratio is used to calculate the fields for $m=0,1$ and 2 , using $\psi_{3}$. This way intensity gain at the boundaries was prevented.

In fig. 1 results of the methods published previously $[2,3]$ are compared with that of the present one. The curves have been calculated using eq. (8), and similar expressions for the other methods. The points have been obtained comparing results of FDBPM simulations, using the present method, with the exact values. The small deviations between the results of eq. (8) and those of the simulations are attributed to the discretisation along the $x$-axis.

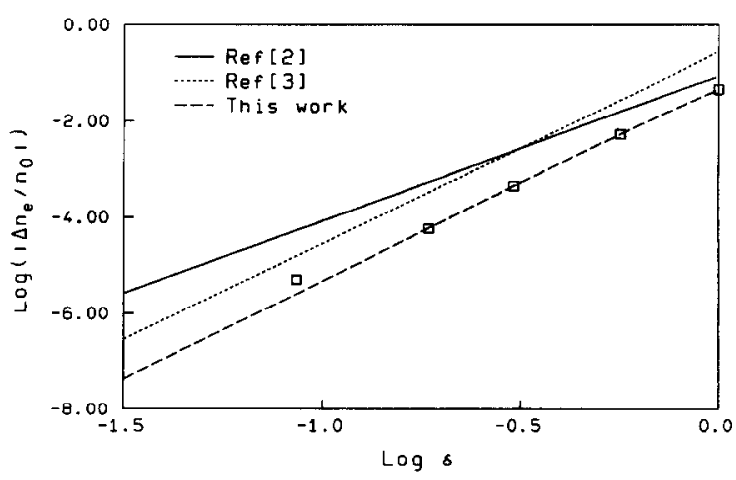

Fig. 1. $\log -\log$ plot of the relative error in the effective index of the fundamental mode with respect to the exact value as a function of $\delta$, obtained using the theoretical expressions for the various methods (see text). For the FDBPM simulations we used a structure consisting of a waveguide with a width, $w=1 \mu \mathrm{m}$, a refractive index, $n_{z}=2$, and the background index $n_{\mathrm{b}}=1$. The stepsize and wavelength are $\Delta x=0.005 \mu \mathrm{m}$ and $\lambda=1 \mu \mathrm{m}$. 


\section{References}

[1] Y. Chung and N. Dagli, IEEE J. Quantum Electron. 26 (1990) 1355.

[2] H.J.W.M. Hoekstra, G.J.M. Krijnen and P.V. Lambeck, Optics Comm. 94 (1992) 506.
[3] G.R. Hadley, Optics. Lett. (1992), to be published.

[4] H.J.W.M. Hoekstra, G.J.M. Krijnen and P.V. Lambeck, J. Lightwave Technol, accepted for publication.

[5] G.R. Hadley, IEEE J. Quantum Electron. 28 ( 1992) 363. 